\title{
Locating and Multiplicative Locating Indices of Graphs with QSPR Analysis
}

\author{
Suha Wazzan $\mathbb{D}^{1}$ and Anwar Saleh $\mathbb{D}^{2}$ \\ ${ }^{1}$ Department of Mathematics, KAU King Abdulaziz University, Science Faculty, 21589 Jeddah, Saudi Arabia \\ ${ }^{2}$ Department of Mathematics, University of Jeddah, Science Faculty, Jeddah, Saudi Arabia \\ Correspondence should be addressed to Suha Wazzan; swazzan@kau.edu.sa
}

Received 21 February 2021; Accepted 26 April 2021; Published 19 May 2021

Academic Editor: Ismail Naci Cangul

Copyright (c) 2021 Suha Wazzan and Anwar Saleh. This is an open access article distributed under the Creative Commons Attribution License, which permits unrestricted use, distribution, and reproduction in any medium, provided the original work is properly cited.

\begin{abstract}
In this paper, by introducing a new version of locating indices called multiplicative locating indices, we compute exact values of these indices on well-known families of graphs and graphs obtained by some operations. Also, we determine the importance of locating and multiplicative locating indices of hexane and its isomers. Furthermore, we show that locating indices actually have a reasonable correlation using linear regression with physico-chemical characteristics such as enthalpy, melting point, and boiling point. This approximation can be extended into several chemical compounds.
\end{abstract}

\section{Introduction}

As an example of a molecular descriptor, a topological graph index is defined as a mathematical formula which is applied to any graph that models some molecular structure. These indices make analyzing mathematical values and examining certain molecules physico-chemical properties more feasible and efficient by enabling us to bypass costly and lengthy laboratory experiments. The role of molecular descriptors is well established in mathematical chemistry. They include but are not limited to $Q S P R$ or quantitative structure-property relationship. There are various topological indices in the literature, and many of them have broad applications in chemistry. The structural properties of the graphs employed in the calculations can be used to classify them. For instance, the Zagreb type indices are computed using the degrees of vertices in a graph. They helped to compare some alkane isomers boiling points and have aided in the discovery, along with other indices, of a few thousand topological graph indices enrolled in the chemical data bases. In fact there has been a rapidly increasing interest of this topic, and thus topological graph indices have been studied worldwide by both mathematicians and chemists (see [1-8]). The most widely known topological indices are the first and second
Zagreb indices, which have been introduced by Gutman and Trinajstic in [9], and defined as $M_{1}(G)=\sum_{u \in V(G)}(d(u))^{2}$ and $M_{2}(G)=\sum_{u v \in E(G)} d(u) d(v)$, respectively. Actually, several new versions of the Zagreb indices have been established for similar purposes (cf. [10-17]).

Different topological indices for some chemical compounds such as "aspirin" and the anticancer drug "carbidopa" have been studied in detail by Wazzan (cf. $[18,19])$. Moreover, in a recent work, Wazzan et al. (see [20]) introduced novel topological indices called the first and second locating indices. To do that, the authors used the locating matrix Lo $(G)$ over a graph $G$ (cf. [21]). Let $G=(V, E)$ be a connected graph with the vertex set $V=\left\{v_{1}, v_{2}, \ldots, v_{n}\right\}$. A locating function of $G$ denoted by $\mathbf{L}(G)$ is a function $\mathbf{L}(G): V(G) \longrightarrow \mathbb{Z}^{+} \cup\{0\}$ such that $\mathbf{L}\left(v_{i}\right)=\overrightarrow{v_{i}}=\left(d\left(v_{1}, v_{i}\right), d\left(v_{2}, v_{i}\right), \ldots, d\left(v_{n}, v_{i}\right)\right)$, where $d\left(v_{i}, v_{j}\right)$ is the distance between the vertices $v_{i}$ and $v_{j}$ in $G$. The vector $\overrightarrow{v_{i}}$ is called the locating vector corresponding to the vertex $v_{i}$, where $\overrightarrow{v_{i}} \cdot \overrightarrow{v_{j}}$ is actually the dot product of the vectors $\overrightarrow{v_{i}}$ and $\overrightarrow{v_{j}}$ in the integers space $\mathbb{Z}^{+} \cup\{0\}$ such that $v_{i}$ is adjacent to $v_{j}$. In the present paper, as a next step of the work in [20], we introduce the first and second multiplicative locating indices for a connected graph $G$ as in the following definition. 
Definition 1. For a connected graph $G=(V, E)$ with an edge set $E(G)$ and vertex set $V=\left\{v_{1}, v_{2}, \ldots, v_{n}\right\}$, the first and second multiplicative locating indices are defined as follows:

$$
\begin{aligned}
& \prod_{1}^{\mathscr{L}}(G)=\prod_{v_{i} \in V(G)}\left(\overrightarrow{v_{i}}\right)^{2}, \\
& \prod_{2}^{\mathscr{L}}(G)=\prod_{v_{i} v_{j} \in E(G)} \overrightarrow{v_{i}} \cdot \overrightarrow{v_{j}},
\end{aligned}
$$

respectively.

In this paper, we only consider simple graphs with no multiple edges. For the terminologies, we may recommend citation [22] to readers.

\section{Certain Values of Multiplicative Locating Indices}

In this section, by considering Definition 1, we will determine the first and second multiplicative locating indices for some special graphs such as $K_{m}, K_{m, n}, C_{m}, W_{m}$, and $P_{m}$, and also we will compute the same indices for the graph $G$ such that $G$ is obtained by joining two graphs $G_{1}$ and $G_{2}$ (notationally $G \cong G_{1}+G_{2}$ ), where $G_{1}$ and $G_{2}$ are connected with diameter 2 . In particular, we will assume that $G$ as $C_{3^{-}}$ and $C_{5}$-free graphs.
Theorem 1. Let $G \cong K_{m}$ be the complete graph with $m \geq 2$. Then,

(1) $\prod_{1}^{\mathscr{L}}\left(K_{m}\right)=(m-1)^{m}$

(2) $\prod_{2}^{\mathscr{L}}\left(K_{m}\right)=(\sqrt{m-2})^{m(m-1)}$

Proof

(i) Let $G \cong K_{m}$ be the complete graph with $m \geq 2$ and let $V(G)=\left\{v_{1}, v_{2}, \ldots, v_{m}\right\}$, and for each vertex $v_{i} \in V(G)$, we let $\overrightarrow{v_{i}}$ is the locating vector associated with the vertex $v_{i}$. Then, $\overrightarrow{v_{i}}=\left(a_{1}, a_{2}, \ldots, a_{m}\right)$ such that $a_{i}=0$ and all the other components are equal to 1. Hence, $\left(\overrightarrow{v_{i}}\right)^{2}=m-1$. However, the total amount of vertices in $G$ is $m$ vertices, and so, $\prod_{1}^{\mathscr{L}}\left(K_{m}\right)=(m-1)^{m}$.

(ii) For any arbitrary locating vectors $\vec{v}_{i}$ and $\vec{v}_{j}$, where $i \neq j$, we gain $\overrightarrow{v_{i}} \cdot \overrightarrow{v_{j}}=m-2$. Therefore, $\prod_{2}^{\mathscr{L}}\left(K_{m}\right)=(\sqrt{m-2})^{m(m-1)}$.

Theorem 2. Let $G \cong K_{m, n}$, where $1 \leq m \leq n$. Then,

(1) $\prod_{1}^{\mathscr{L}}\left(K_{m, n}\right)=(4 m-4+n)^{m}+(4 n-4+m)^{n}$

(2) $\prod_{2}^{\mathscr{L}}\left(K_{m, n}\right)=(2 m+2 n-4)^{n m}$

Proof. We identify the adjacent vertices $v_{i}$ and $v_{m+j}$ of $K_{m, n}$, for all $1 \leq j \leq n$ and $1 \leq i \leq m$. Then, the locating vectors $\overrightarrow{v_{i}}$ of $v_{i}$ are given by

$$
\begin{aligned}
& \overrightarrow{v_{1}}=(0, \overbrace{2, \ldots, 2}^{m-1}, \overbrace{1,1, \ldots, 1}^{n}), \vec{v}_{2}=(2,0, \overbrace{2, \ldots, 2}^{m-2}, \overbrace{1,1, \ldots, 1}^{n}) \\
& \overrightarrow{v_{3}}=(2,2,0, \overbrace{2, \ldots, 2}^{m-3}, \overbrace{1,1, \ldots, 1}^{n}), \quad \ldots, \quad \overrightarrow{v_{m}}=(\overbrace{2, \ldots, 2}^{m-1}, 0, \overbrace{1,1, \ldots, 1}^{n}), \\
& \underset{v_{m+1}}{\longrightarrow}=(\overbrace{1, \ldots, 1}^{m}, 0, \overbrace{2,2, \ldots, 2}^{n-1}), \quad \vec{v}_{m+2}=(\overbrace{1, \ldots, 1}^{m}, 2,0, \overbrace{2, \ldots, 2}^{n-2}) . \quad \vdots \quad \vdots \quad \vec{v}_{m+n}=(\overbrace{1, \ldots, 1}^{n}, \overbrace{2, \ldots, 2}^{m-1}, 0) . \\
& \prod_{1}^{\mathscr{L}}\left(K_{m, m}\right)=2(5 m-4)^{m}, \prod_{2}^{\mathscr{L}}\left(K_{m, n}\right)=(4 m-4)^{m^{2}} .
\end{aligned}
$$
$\vec{v}_{i}^{2}=4(n-1)+m$. Therefore,

$$
\begin{aligned}
\prod_{1}^{\mathscr{L}}\left(K_{m, n}\right) & =(4(m-1)+n)^{m}+(4(n-1)+m)^{n} \\
& =(4 m-4+n)^{m}+(4 n-4+m)^{n} .
\end{aligned}
$$

Similarly, for any two locating vertices $\vec{v}, \vec{u}$ in $K_{m, n}$ where $u$ adjacent to $v, \vec{v} \cdot \vec{u}=2(m+n-2)$. Hence, $\prod_{2}^{\mathscr{L}}\left(K_{m, n}\right)=(2 m+2 n-4)^{n m}$.

Corollary 1. Let $G \cong K_{m, m}$, where $m \geq 1$. Then,
Corollary 2. Let $G$ be any star graph $K_{1, n}$. Then,

$$
\prod_{1}^{\mathscr{L}}\left(K_{1, n}\right)=n+(4 n+3)^{n}, \prod_{2}^{\mathscr{L}}\left(K_{1, n}\right)=(2 n-2)^{n} .
$$

Theorem 3. For an even integer $m \geq 2$, let $G \cong C_{m}$. Then,

$$
\prod_{1}^{\mathscr{L}}\left(C_{m}\right)=\left(\frac{m^{3}+2 m}{12}\right)^{m}, \prod_{2}^{\mathscr{L}}\left(C_{m}\right)=\left(\frac{m(m-2)^{2}}{12}\right)^{m} \text {. }
$$


Proof. By identifying the vertices of the cycle $C_{m}$ as $\left\{v_{1}, v_{2}, \ldots, v_{m}\right\}$ in the anticlockwise direction, we obtain

$$
\begin{aligned}
\overrightarrow{v_{1}} & =\left(0,1,2,3, \ldots, \frac{m}{2}, \frac{m}{2}-1, \frac{m}{2}-2, \ldots, 1\right), \\
\overrightarrow{v_{2}} & =\left(1,0,1,2, \ldots, \frac{m}{2}-1, \frac{m}{2}, \frac{m}{2}-1, \ldots, 2\right), \\
\overrightarrow{v_{3}} & =\left(2,1,0,1, \ldots, \frac{m}{2}-2, \frac{m}{2}-1, \frac{m}{2}, \ldots, 3\right), \\
\vdots & \quad \vdots \\
\overrightarrow{v_{m}} & =\left(1,2,3, \ldots, \frac{m}{2}, \frac{m}{2}-1, \frac{m}{2}-2, \frac{m}{2}-2, \ldots, 0\right),
\end{aligned}
$$

and hence $\vec{v}_{i}^{2}=2\left(\sum_{i=1}^{(m / 2)} i^{2}\right)-\left(m^{2} / 4\right)$. It is straightforward to see that each $\overrightarrow{v_{i}}$ has equivalent components but in different locations; hence, each $\vec{v}_{i}^{2}$ has the same sum as the form of

$$
{\overrightarrow{v_{i}}}^{2}=\frac{m(m+1)(m+2)-3 m^{2}}{12}
$$

Therefore, $\prod_{2}^{\mathscr{L}}\left(C_{m}\right)=\left(m^{3}+2 m / 12\right)^{m}$. By symmetry,

$$
\overrightarrow{v_{i}} \cdot \overrightarrow{v_{i+1}}=2 \sum_{i=2}^{(m / 2)} i(i-1)=2\left(\frac{(m / 2)((m / 2)+1)(m+1)}{6}-1\right)-2\left(\frac{(m / 2)((m / 2)+1)}{2}-1\right)=\frac{m(m-2)^{2}}{12}
$$

which gives $\prod_{2}^{\mathscr{L}}\left(C_{m}\right)=\left(m(m-2)^{2} / 12\right)^{m}$.

$$
\prod_{1}^{\mathscr{L}}\left(C_{m}\right)=\left(\frac{m\left(m^{2}-1\right)}{12}\right)^{m}, \prod_{2}^{\mathscr{L}}\left(C_{m}\right)=\left(\frac{(m-1)(m-2)(m+3)}{12}\right)^{m} .
$$

Proof. Following the steps in the proof of Theorem 4, we get

$$
\begin{gathered}
\overrightarrow{v_{1}}=\left(0,1,2,3, \ldots, \frac{m-1}{2}, \frac{m-1}{2}-1, \frac{m-1}{2}-2, \ldots, 1\right), \\
\overrightarrow{v_{2}}=\left(1,0,1,2, \ldots, \frac{m-1}{2}-1, \frac{m-1}{2}, \frac{m-1}{2}-1, \ldots, 2\right), \\
\overrightarrow{v_{3}}=\left(2,1,0,1, \ldots, \frac{m-1}{2}-2, \frac{m-1}{2}-1, \frac{m-1}{2}, \ldots, 3\right), \\
\vdots \quad \vdots \\
\overrightarrow{v_{m}}=\left(1,2,3, \ldots, \frac{m-1}{2}, \frac{m-1}{2}-1, \frac{m-1}{2}-2, \ldots, 0\right),
\end{gathered}
$$

Theorem 4. Let $G \cong C_{m}$ with an odd number of vertices $m \geq 3$. Then,

$$
\begin{aligned}
\overrightarrow{v_{i}} \cdot \overrightarrow{v_{i+1}}= & 2 \sum_{i=2}^{(m-1 / 2)} i(i-1)+\frac{(m-1)^{2}}{4} \\
= & \left(2 \frac{(m-1 / 2)((m-1 / 2)+1)(2(m-1 / 2)+1)}{6}\right)-1-\left(2 \frac{(m-1 / 2)((m-1 / 2)+1)}{2}-1\right) \\
& +\frac{(m-1)^{2}}{4}=\frac{(m-1)(m-2)(m+3)}{12} .
\end{aligned}
$$

Hence, $\prod_{2}^{\mathscr{L}}\left(C_{m}\right)=((m-1)(m-2)(m+3) / 12)^{m}$, as required.

$$
\prod_{1}^{\mathscr{L}}\left(W_{m}\right)=m(4 m-9)^{m}, \prod_{2}^{\mathscr{L}}\left(W_{m}\right)=\left(8 m^{2}-38 m+44\right)^{m}
$$

which implies $\prod_{2}^{\mathscr{L}}\left(C_{m}\right)=\left(m\left(m^{2}-1\right) / 12\right)^{m}$. Further, by the symmetry,

Theorem 5. Let $G$ be wheel graph $W_{m}$ with $m+1$ vertices such that $(m \geq 4)$. Then, 
Proof. Let $G \cong W_{m}$ with $m+1$ vertices. Suppose that the vertices $v_{1}, v_{2}, \ldots, v_{m}, v_{m+1} \in V(G)$ are labeling in the anticlockwise direction where the center of the wheel is labeled $v_{m+1}$. Hence, we get

$$
\overrightarrow{v_{1}}=(0,1, \overbrace{2,2, \ldots, 2}^{m-3}, 1,1), \overrightarrow{v_{2}}=(1,0,1, \overbrace{2,2, \ldots, 2}^{m-3}, 1), \overrightarrow{v_{3}}=(2,1,0,1, \overbrace{2, \ldots, 2}^{m-2}, 1), \ldots, \overrightarrow{v_{m}}=(1, \overbrace{2,2, \ldots, 2}^{m-3}, 1,0,1), \overrightarrow{v_{m+1}}=(\overbrace{1,1, \ldots, 1}^{m}, 0) .
$$

Therefore, for each corresponding locating vector $\overrightarrow{v_{i}}$ with the vertex $v_{i}(i \in\{1,2, \ldots, m\})$, we have ${\overrightarrow{v_{i}}}^{2}=4 m-9$ and $\overrightarrow{v_{m+1}}=m$. So, $\prod_{1}^{\mathscr{L}}\left(W_{m}\right)=m(4 m-9)^{m}$. For
$\prod_{2}^{\mathscr{L}}\left(W_{m}\right)$, by considering the same labeling as previously, we get

$$
\begin{aligned}
& \overrightarrow{v_{1}}=(\mathbf{0}, \mathbf{1}, \underbrace{2,2, \ldots, 2}_{m-3}, 1, \mathbf{1}), \overrightarrow{v_{2}}=(\mathbf{1}, \mathbf{0}, \mathbf{1}, \underbrace{2,2, \ldots, 2}_{m-3}, 1), \overrightarrow{v_{3}}=(2, \mathbf{1}, \mathbf{0}, \mathbf{1}, \underbrace{2, \ldots, 2}_{m-2}, 1), \overrightarrow{v_{4}}=(2,2, \mathbf{1}, \mathbf{0}, \mathbf{1}, \underbrace{2, \ldots, 2}_{m-1}, 1), \\
& \overrightarrow{v_{5}}=(2,2,2, \mathbf{1}, \mathbf{0}, \mathbf{1}, \underbrace{2, \ldots, 2}_{m}, 1), \ldots, \overrightarrow{v_{m}}=(1, \underbrace{2,2, \ldots, 2}_{m-3}, \mathbf{1}, \mathbf{0}, \mathbf{1}), \overrightarrow{v_{m+1}}=(\underbrace{1,1, \ldots, 1}_{m}, 0) .
\end{aligned}
$$

Here, the permutation components in each vector $\overrightarrow{v_{i}}$ where $i=1,2, \ldots, m$ are $\mathbf{1}, \mathbf{0}, \mathbf{1}$. Hence, it is straightforward to notice that any two adjacent vertices $v_{i}$ and $v_{j}(i, j \in\{1,2, \ldots, m\})$ satisfy $\overrightarrow{v_{i}} \cdot \overrightarrow{v_{j}}=4 m-11$ and $\overrightarrow{v_{i}} \cdot \overrightarrow{v_{m+1}}=2 m-4$ for $i=1,2, \ldots, m$. Therefore,

$\prod_{2}^{\mathscr{L}}\left(W_{m}\right)=\left(8 m^{2}-38 m+44\right)^{m}$. Hence, the result is obtained.

Theorem 6. For any path $P_{m}$ with $(m \geq 3)$ vertices,

$$
\prod_{1}^{\mathscr{L}}\left(P_{m}\right)=\frac{(m-j)(m-j+1)(2 m-2 j+1)+j(j-1)(2 j-1)}{6}, \prod_{2}^{\mathscr{L}}\left(P_{m}\right)=\prod_{j=1}^{m-1} \frac{(m-j)(m-j+1)(m-j-1)}{3} .
$$

Proof. Assume that $P_{m}$ is the path with $(m \geq 3)$ vertices. Suppose that the locating function is constructed by identifying the vertices as $v_{1}, v_{2}, \ldots, v_{m}$ from left to right. Hence, the corresponding vectors for each vertex $v_{i} \in V(G)(i=$ $1, \ldots, m)$ are given as in following:

$\overrightarrow{v_{1}}=(0,1,2,3, \ldots, m-1), \overrightarrow{v_{2}}=(1,0,1,2, \ldots, m-2), \ldots, \overrightarrow{v_{m-1}}=(m-2, m-1, \ldots, 0,1), \overrightarrow{v_{m}}=(m-1, m-2, m-3, \ldots, 0)$

A straightforwardly calculation implies that

$$
\prod_{1}^{\mathscr{L}}\left(P_{m}\right)=\prod_{j=1}^{m}\left(\sum_{i=1}^{m-j} i^{2}+\sum_{i=1}^{j-1} i^{2}\right)=\prod_{j=1}^{m} \frac{(m-j)(m-j+1)(2 m-2 j+1)+j(j-1)(2 j-1)}{6} .
$$

For the other case $\prod_{2}^{\mathscr{L}}\left(P_{m}\right)$, 


$$
\begin{aligned}
& \vec{v}_{1} \cdot \vec{v}_{2}=(0 \cdot 1)+(1 \cdot 0)+\cdots+(m-1)(m-2)=\sum_{i=1}^{m-1} i(i-1), \\
& \vec{v}_{2} \cdot \vec{v}_{3}=(1 \cdot 2)+(0 \cdot 1)+\cdots+(m-2)(m-3)=\sum_{i=1}^{m-1} i(i-1), \\
& \vec{v}_{3} \cdot \vec{v}_{4}=\sum_{i=1}^{m-1} i(i-1) .
\end{aligned}
$$

So, we get

$$
\prod_{2}^{\mathscr{L}}\left(P_{m}\right)=\prod_{j=1}^{m-1} \sum_{i=1}^{m-j} i(i-1)=\prod_{j=1}^{m-1}\left(\sum_{i=1}^{m-j} i^{2}-\sum_{i=1}^{m-j} i\right) .
$$

Therefore, $\prod_{2}^{\mathscr{L}}\left(P_{m}\right)$ is obtained as required in the statement of theorem.

In the following result, we will give our attention to the join $G \cong G_{1}+G_{2}$ of graphs $G_{1}$ and $G_{2}$ for computing multiplicative locating indices.

Theorem 7. Let $G \cong G_{1}+G_{2}$ such that $G_{1}$ and $G_{2}$ are both connected graphs, where $G_{1}$ and $G_{2}$ have $m_{1}$ edges; $n_{1}$ vertices and $m_{2}$ edges $n_{2}$ vertices, respectively. Then,

$$
\prod_{1}^{\mathscr{L}}(G)=\prod_{i=1}^{n_{1}}\left(n_{2}+4 n_{1}-4-3 \operatorname{deg}_{G_{1}}\left(v_{i}\right)\right) \prod_{j=n_{1}+1}^{n_{1}+n_{2}}\left(n_{1}+4 n_{2}-4-3 \operatorname{deg}_{G_{2}}\left(v_{j}\right)\right) .
$$

Proof. Let $G$ be as in the statement of theorem. Let us label the vertices of the graph $G$ as

$$
v_{1}, v_{2}, \ldots, v_{n_{1}}, v_{n_{1}+1}, v_{n_{1}+2}, \ldots, v_{n_{1}+n_{2}},
$$

where $v_{1}, v_{2}, \ldots, v_{n_{1}} \in V\left(G_{1}\right)$ and $v_{n_{1}+1}, v_{n_{1}+2}, \ldots, v_{n_{1}+n_{2}} \in$ $V\left(G_{2}\right)$. In addition, suppose that

$$
\vec{v}=(0, \underbrace{1, \ldots, 1}_{\operatorname{deg}_{G_{1}}(v)}, \underbrace{2, \ldots, 2}_{n_{1}-\operatorname{deg}_{G_{1}}(v)-1}, \underbrace{1, \ldots, 1}_{n_{2}}),
$$

which is the locating vector associated with the vertex $v \in V\left(G_{1}\right)$. Then, $\vec{v}^{2}=n_{2}+4 n_{1}-4-3 \operatorname{deg}_{G_{1}}(v)$. Similarly, for any vertex $z \in V\left(G_{2}\right)$, the locating vector $\vec{z}$ corresponding to $z$ is given by

$$
\vec{z}=(\underbrace{1, \ldots, 1}_{n_{1}}, 0, \underbrace{1, \ldots, 1}_{\operatorname{deg}_{G_{2}}(z)}, \underbrace{2, \ldots, 2}_{n_{2}-\operatorname{deg}_{G_{2}}(z)-1}) \text {. }
$$

So, $\vec{z}^{2}=n_{1}+4 n_{2}-4-3 \operatorname{deg}_{G_{2}}(z)$. Therefore, by the above equalities on $\vec{v}^{2}$ and $\vec{z}^{2}$, we obtain $\prod_{1}^{\mathscr{L}}(G)$ as required in the theorem.

Theorem 8. Suppose that $G_{1}$ and $G_{2}$ are connected graphs having diameter 2. Let $G \cong G_{1}+G_{2}$ such that $G$ is a $C_{3}$-or $C_{5}$-free graph. Assume that $G_{1}$ has $m_{1}$ edges and $n_{1}$ vertices while $G_{2}$ has $m_{2}$ edges and $n_{2}$ vertices. Then,

$$
\prod_{2}^{\mathscr{L}}(G)=\alpha \beta
$$

$$
\begin{aligned}
& \alpha=\left(n_{2}+2 n_{1}-4\right)^{m_{1}}\left(n_{1}+2 n_{2}-4\right)^{m_{2}}, \\
& \beta=\prod_{\substack{u \in V\left(G_{1}\right), v \in V\left(G_{2}\right), u v \in E(G)}}\left(2\left(n_{1}+n_{2}\right)-4-\left(\operatorname{deg}_{G_{1}}(u)-\operatorname{deg}_{G_{2}}(v)\right)\right)^{n_{1} n_{2}} .
\end{aligned}
$$

Proof. Under the assumptions on $G$ as in the statement of the theorem, the partition sets edges are defined by

$$
\begin{aligned}
& A=\left\{u v: u, v \in V\left(G_{1}\right)\right\}, \\
& B=\left\{u v: u, v \in V\left(G_{2}\right)\right\}, \\
& C=\left\{u v: u \in V\left(G_{1}\right), v \in V\left(G_{2}\right)\right\} .
\end{aligned}
$$

Hence, $\prod_{2}^{\mathscr{L}}(G)$ is expressed as

$$
\prod_{u v \in A} \vec{u} \cdot \vec{v} \prod_{u v \in B} \vec{u} \cdot \vec{v} \prod_{u v \in C} \vec{u} \cdot \vec{v}
$$

For any two adjacent vertices $u, v \in V\left(G_{1}\right)$ to obtain $\prod_{u v \in A} \vec{u} \cdot \vec{v}$, we assume that the first two vertices as follows:

$$
\begin{aligned}
& \vec{u}=(0, \underbrace{1, \ldots, 1}_{\operatorname{deg}_{G_{1}}(u)}, \underbrace{2, \ldots, 2}_{n_{1}-\operatorname{deg}_{G_{1}}(u)-1}, \underbrace{1, \ldots, 1}_{n_{2}}), \\
& \vec{v}=(1,0, \underbrace{2, \ldots, 2}_{\operatorname{deg}_{G_{1}}(u)-1}, \underbrace{1, \ldots, 1}_{n_{1}-\operatorname{deg}_{G_{1}}(u)-1}, \underbrace{1, \ldots, 1}_{n_{2}}) .
\end{aligned}
$$

Since $G_{1}$ and $G_{2}$ are $C_{3}$ - or $C_{5}$-free graph, for any two vertices $u$ and $v$ in $V\left(G_{1}\right)$, we can obtain

where

$$
\vec{u} \cdot \vec{v}=2\left(\operatorname{deg}_{G_{1}}(u)-1\right)+2\left(n_{1}-\operatorname{deg}_{G_{1}}(u)-1\right)+n_{2}=n_{2}+2 n_{1}-4
$$


which implies $\prod_{u \cdot v \in A} \vec{u} \cdot \vec{v}=\left(n_{2}+2 n_{1}-4\right)^{m_{1}}$.

With the same way of calculation, we get As a result, we get $\prod_{u \cdot v \in B} \vec{u} \cdot \vec{v}=\left(n_{1}+2 n_{2}-4\right)^{m_{2}}$. Now, to achieve the computation of $\prod_{u v \in C} \vec{u} \cdot \vec{v}$, let us take $u \in V\left(G_{1}\right)$ and $v \in V\left(G_{2}\right)$. Thus,

$$
\begin{aligned}
& \vec{u}=(0, \underbrace{1, \ldots, 1}_{\operatorname{deg}_{G_{1}}(u)}, \underbrace{2, \ldots, 2}_{n_{1}-\operatorname{deg}_{G_{1}}(u)-1}, \underbrace{1, \ldots, 1}_{n_{2}}) . \\
& \vec{v}=(\underbrace{1, \ldots, 1}_{\log _{G_{1}}(u)+1}, \underbrace{1, \ldots, 1}_{n_{1}-\operatorname{deg}_{G_{1}}(u)-1}, 0, \underbrace{1, \ldots, 1}_{\operatorname{deg}_{G_{2}}(v)}, \underbrace{2, \ldots, 2}_{n_{2}-\operatorname{deg}_{G_{2}}(v)-1}) .
\end{aligned}
$$

$$
\begin{aligned}
\vec{u} \cdot \vec{v} & =\operatorname{deg}_{G_{1}}(u)+2\left(n_{1}-\operatorname{deg}_{G_{2}}(u)-1\right)+\operatorname{deg}_{G_{2}}(v)+2\left(n_{2}-\operatorname{deg}_{G_{2}}(v)-1\right) \\
& =2\left(n_{1}+n_{2}\right)-4-\left(\operatorname{deg}_{G_{1}}(u)-\operatorname{deg}_{G_{2}}(v)\right),
\end{aligned}
$$

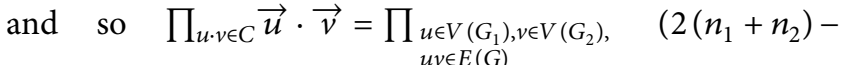
$\left.4-\left(\operatorname{deg}_{G_{1}}(u)-\operatorname{deg}_{G_{2}}(v)\right)\right)^{n_{1} n_{2}}$. Then, by all above calculations, we finally get $\prod_{2}^{\mathscr{L}}(G)=\alpha \beta$, where

$$
\begin{aligned}
& \alpha=\left(n_{2}+2 n_{1}-4\right)^{m_{1}}\left(n_{1}+2 n_{2}-4\right)^{m_{2}}, \\
& \beta=\prod_{\substack{u \in V\left(G_{1}\right), v \in V\left(G_{2}\right), u v \in E(G)}}\left(2\left(n_{1}+n_{2}\right)-4-\left(\operatorname{deg}_{G_{1}}(u)-\operatorname{deg}_{G_{2}}(v)\right)\right)^{n_{1} n_{2}} .
\end{aligned}
$$

Hence, the result is obtained.
Theorem 9. Let $G$ be a book graph $B_{t}=P_{2} \times S_{t}$ with $2 t+2$ vertices. Then,

$$
\prod_{1}^{\mathscr{L}}(G)=(5 t+1)^{2}(13 t-7)^{2 t}, \prod_{2}^{\mathscr{L}}(G)=\left(4 t(12 t-8)(8 t+4)^{2}\right)^{t} .
$$

Proof. Let us label the vertices $V(G)=\left\{v, u, v_{1}, v_{2}, \ldots v_{t}, u_{1}, u_{2}, \ldots, u_{t}\right\}$ as in Figure 1. So, we have

$$
\begin{aligned}
& \vec{v}=(0,1, \overbrace{1,1, \ldots, 1}^{t}, \overbrace{2,2, \ldots, 2}^{t}, \vec{u}=(1,0, \overbrace{2,2, \ldots, 2}^{t}, \overbrace{1,1, \ldots, 1}^{t}) \\
& \overrightarrow{v_{1}}=(1,2,0, \overbrace{2, \ldots, 2}^{t-1}, 1, \overbrace{3, \ldots, 3}^{t-1}) \text {, } \\
& \overrightarrow{u_{1}}=(2,1,1, \overbrace{3, \ldots, 3}^{t-1}, 0, \overbrace{2, \ldots, 2}^{t-1}) \\
& \overrightarrow{v_{2}}=(1,2,2,0, \overbrace{2, \ldots, 2}^{t-2}, 3,1, \overbrace{3, \ldots, 3}^{t-2}), \\
& \overrightarrow{u_{2}}=(, 1,3,1, \overbrace{3, \ldots, 3}^{t-2}, 2,0, \overbrace{2, \ldots, 2}^{t-2}) \\
& \vdots \quad \vdots \\
& \overrightarrow{v_{t}}=(1,2, \overbrace{2, \ldots, 2}^{t-1}, 0, \overbrace{3, \ldots, 3}^{t-1}, 1), \quad \vec{u}_{t}=(2,1, \overbrace{3, \ldots, 3}^{t-1}, 1, \overbrace{2, \ldots, 2}^{t-1}, 0) .
\end{aligned}
$$

Considering the components of the locating vectors of the book graph, we get $\vec{v}^{2}=\vec{u}^{2}=(t+1)+4 t=5 t+1$, and for $i=1,2,3, \ldots, t$, we have $\vec{v}_{i}^{2}={\overrightarrow{u_{i}}}^{2}=(13 t-7)$. Hence,
$\prod_{1}^{\mathscr{L}}(G)=(5 t+1)^{2}(13 t-7)^{2 t}$. Similarly, we have $\vec{v} \cdot \vec{u}=4 t$, and for any $i=1,2,3, \ldots, t$, $\overrightarrow{v_{i}} \cdot \overrightarrow{u_{i}}=4+6(t-1)+6(t-1)=12 t-8$, and in the same 


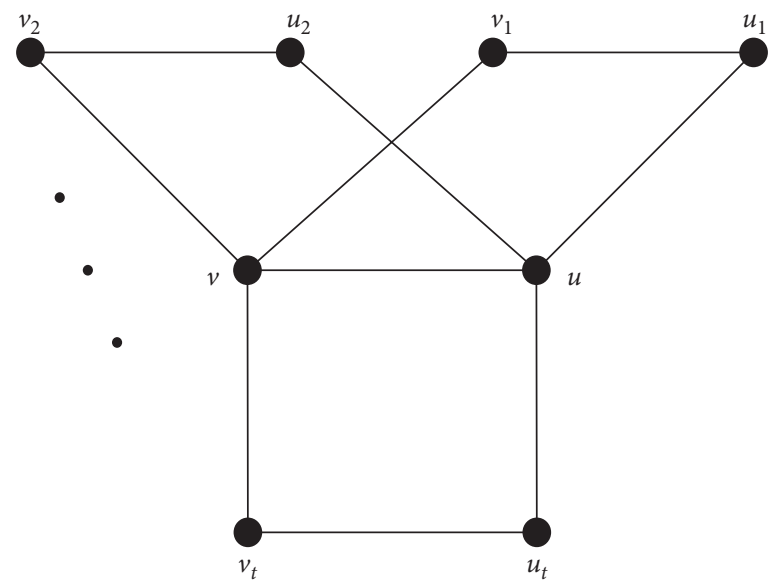

FIgURE 1: A book graph $B_{t}$.

way, $\vec{v} \cdot \overrightarrow{v_{i}}=\vec{u} \cdot \overrightarrow{u_{i}}=8 t-4$. Hence, $\prod_{2}^{\mathscr{L}}(G)=4 t((12 t-$ 8) $\left.(8 t-4)^{2}\right)^{t}$.

\section{Multiplicative Locating Indices of Firefly Graphs}

A firefly graph $F_{s, t, n-2 s-2 t-1} \quad(s \geq 0, \quad t \geq 0$, and $n-2 s-2 t-1 \geq 0)$ is a graph of order $n$ that consists of $s$ triangles, $t$ pendant paths of length 2 , and $n-2 s-2 t-1$ pendent edges that are sharing a common vertex [23]. Let $\mathscr{F}_{n}$ be the set of all firefly graphs $F_{s, t, n-2 s-2 t-1}$. Note that $\mathscr{F}_{n}$ contains the stars $S_{n}\left(\cong F_{0,0, n-1}\right)$, stretched stars $\left(\cong F_{0, t, n-2 t-1}\right)$, friendship graphs $\left(\cong F_{(n-1 / 2), 0,0}\right)$, and butterfly graphs $\left(\cong F_{s, 0, n-2 s-1}\right)$.

In the following result, the first and second multiplicative locating indices for the firefly graph are calculated. To simplify the calculations, let us denote $n-2 s-2 t-1$ by $l$.

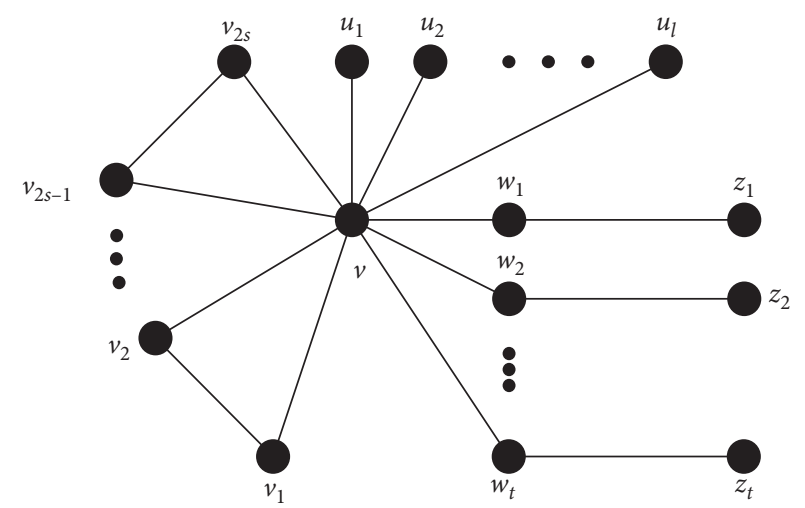

Figure 2: Firefly graph $F_{s, t, l}$.

Theorem 10. Let $G \cong F_{s, t, l}(s, t, l \geq 0)$ be a firefly graph of order $n$. Then,

$$
\begin{aligned}
& \prod_{1}^{\mathscr{L}}(G)=(2 s+5 t+l)^{2}(8 s+13 t+4 l-6)^{2 s}(8 s+13 t+4 l-3)^{l}(8 s+13 t+4 l-11)^{t}(18 s+25 t+9 l-20)^{t} \\
& \prod_{2}^{\mathscr{L}}(G)=(8 s+13 t+4 l-7)^{2 s-1}(4 s+8 t+2 l-3)^{2 s}(4 s+8 t+2 l-2)^{l}(4 s+8 t+2 l-6)^{t}(12 s+8 t+6 l-16)^{t}
\end{aligned}
$$

Proof. Suppose that $G \cong F_{s, t, l}(s, t, l \geq 0)$ is a firefly graph of $n=2 s+2 t+l+1$ vertices. Let us label the vertices of the graph (see Figure 2) with clockwise direction.

So, in the set

$V(G)=\left\{v, v_{1}, v_{2}, \ldots v_{2 s}, u_{1}, u_{2}, \ldots, u_{l}, w_{1}, w_{2}, \ldots, w_{t}, z_{1}, z_{2}, \ldots, z_{t}\right\}$, where $v$ is the center vertex of the firefly graph, $v_{1}, \ldots v_{2 s}$ is the vertices of the triangles, $u_{1}, \ldots u_{l}$ is the vertices of the pendent edges, $w_{1}, \ldots, w_{t}$ is the first vertices of the pendent paths, and $z_{1}, \ldots, z_{t}$ be the second vertices of the pendent paths. Therefore, we obtain the corresponding vectors $\vec{v}_{i}$ for each vertex $v_{i} \in V(G)$ where $i=1,2, \ldots, 2 s+2 t+l+1$ as follows: 


$$
\begin{aligned}
& \vec{v}=(0, \underbrace{1,1, \ldots, 1}_{2 s}, \underbrace{1,1, \ldots, 1}_{l}, \underbrace{1,1, \ldots, 1}_{t}, \underbrace{2,2, \ldots, 2}_{t}), \\
& \vec{v}_{1}=(1,0,1, \underbrace{2,2, \ldots, 2}_{2 s-2}, \underbrace{2,2, \ldots, 2}_{l}, \underbrace{2,2, \ldots, 2}_{t}, \underbrace{3,3, \ldots, 3}_{t}) \text {, } \\
& \vec{v}_{2}=(1,1,0, \underbrace{2,2, \ldots, 2}_{2 s-2}, \underbrace{2,2, \ldots, 2}_{l}, \underbrace{2,2, \ldots, 2}_{t}, \underbrace{3,3, \ldots, 3}_{t}) \text {, } \\
& \vec{v}_{3}=(1,2,2,0,1, \underbrace{2,2, \ldots, 2}_{2 s-4}, \underbrace{2,2, \ldots, 2}_{l}, \underbrace{2,2, \ldots, 2}_{t}, \underbrace{3,3, \ldots, 3}_{t}) \text {, } \\
& \vec{v}_{2 s-1}=(1, \underbrace{2,2, \ldots, 2}_{2 s-2}, 0,1, \underbrace{2,2, \ldots, 2}_{l}, \underbrace{2,2, \ldots, 2}_{t}, \underbrace{3,3, \ldots, 3}_{t}) \text {, } \\
& \begin{array}{c}
\vec{v}_{2 s} \\
\quad:
\end{array} \\
& \vec{u}_{1}=(1, \underbrace{2,2, \ldots, 2}_{2 s}, 0, \underbrace{2,2, \ldots, 2}_{l-1}, \underbrace{2,2, \ldots, 2}_{t}, \underbrace{3,3, \ldots, 3}_{t}), \\
& \vec{u}_{2}=(1, \underbrace{2,2, \ldots, 2}_{2 s}, 2,0, \underbrace{2,2, \ldots, 2}_{l-2}, \underbrace{2,2, \ldots, 2}_{t}, \underbrace{3,3, \ldots, 3}_{t}), \\
& \vec{u}_{l}=(1, \underbrace{2,2, \ldots, 2}_{2 s}, \underbrace{2,2, \ldots, 2}_{l-1}, 0, \underbrace{2,2, \ldots, 2}_{t}, \underbrace{3,3, \ldots, 3}_{t}), \\
& \vec{w}_{1}=(1, \underbrace{2,2, \ldots, 2}_{2 s}, \underbrace{2,2, \ldots, 2}_{l}, 0, \underbrace{2,2, \ldots, 2}_{t-1}, 1, \underbrace{3,3, \ldots, 3}_{t-1}) \text {, } \\
& \vec{w}_{2}=(1, \underbrace{2,2, \ldots, 2}_{2 s}, \underbrace{2,2, \ldots, 2}_{l}, 2,0, \underbrace{2,2, \ldots, 2}_{t-2}, 3,1, \underbrace{3,3, \ldots, 3}_{t-2}) \text {, } \\
& \vec{w}_{t}=(1, \underbrace{2,2, \ldots, 2}_{2 s}, \underbrace{2,2, \ldots, 2}_{l}, \underbrace{2,2, \ldots, 2}_{t-1}, 0, \underbrace{3,3, \ldots, 3}_{t-1}, 1), \\
& \vec{z}_{1}=(2, \underbrace{3,3, \ldots, 3}_{2 s}, \underbrace{3,3, \ldots, 3}_{l}, 1, \underbrace{3,3, \ldots, 3}_{t-1}, 0, \underbrace{4,4, \ldots, 4}_{t-1}), \\
& \vec{z}_{2}=(2, \underbrace{3,3, \ldots, 3}_{2 s}, \underbrace{3,3, \ldots, 3}_{l}, 3,1, \underbrace{3,3, \ldots, 3}_{t-2}, 4,0, \underbrace{4,4, \ldots, 4}_{t-2}) \text {, } \\
& \vec{z}_{t}=(2, \underbrace{3,3, \ldots, 3}_{2 s}, \underbrace{3,3, \ldots, 3}_{l}, \underbrace{3,3, \ldots, 3}_{t-1}, 1, \underbrace{4,4, \ldots, 4}_{t-1}, 0) .
\end{aligned}
$$

Obviously,

Hence, we obtain the equality in (38).

$$
\prod_{1}^{\mathscr{L}}(G)=\vec{v}^{2} \prod_{i=1}^{2 s} \vec{v}_{i}^{2} \prod_{i=1}^{l} \vec{u}_{i}^{2} \prod_{i=1}^{t} \vec{w}_{i}^{2} \prod_{i=1}^{t} \vec{z}_{i}^{2}
$$

$$
\prod_{1}^{\mathscr{L}}(G)=(2 s+5 t+l)^{2}(8 s+13 t+4 l-6)^{2 s}(8 s+13 t+4 l-3)^{l}(8 s+13 t+4 l-11)^{t}(18 s+25 t+9 l-20)^{t}
$$


Similarly, as in the above process, since

$$
\prod_{2}^{\mathscr{L}}(G)=\prod_{i=1}^{2 s-1} \overrightarrow{v_{i}} \cdot \overrightarrow{v_{i+1}} \prod_{i=1}^{2 s} \vec{v} \cdot \overrightarrow{v_{i}} \prod_{i=1}^{l} \vec{v} \cdot \overrightarrow{u_{i}} \prod_{i=1}^{t} \vec{v} \cdot \overrightarrow{w_{i}} \prod_{i=1}^{t} \overrightarrow{w_{i}} \cdot \overrightarrow{z_{i}}
$$

$$
\prod_{1}^{\mathscr{L}}(G)=(n-1)^{2}(4 n-10)^{n-1}, \prod_{2}^{\mathscr{L}}(G)=(4 n-11)^{n-2}(2 n-5)^{n-1} .
$$

we get the equality in (39) as required.

\section{Corollary 3}

(1) For any friendship graph of $n$ vertices,
(2) For any butterfly graph $F_{s, 0, n-2 s-1}$ of $n$ vertices,

$$
\prod_{1}^{\mathscr{L}}(G)=(n-1)^{2}(4 n-10)^{2 s}(4 n-7)^{n-2 s-1}, \prod_{2}^{\mathscr{L}}(G)=(4 n-9)^{2 s-1}(2 n-5)^{2 s}(2 n-4)^{n-2 s-1} .
$$

\section{Locating and Multiplicative Locating Indices of Hexane and Its Isomers}

In this section, we will compute some first and second locating and multiplicative indices for hexane and its isomers. Recall that the first and second locating indices [20] are defined as follows:

$$
\begin{aligned}
& M_{1}^{\mathscr{L}}(G)=\sum_{v_{i} \in V}\left(\vec{v}_{i}\right)^{2}, \\
& M_{2}^{\mathscr{L}}(G)=\sum_{v_{i} v_{j} \in E} \overrightarrow{v_{i}} \cdot \overrightarrow{v_{j}} .
\end{aligned}
$$

Hexane and its four structural isomers, namely, 2methylpentane, 3-methylpentane, 2,2-dimethylbutane, and 2,3-dimethylbutane, were fully optimized free of any structural constrains using the one of the well-known functional of the density functional theory (DFT), i.e., B3LYP. B3LYP stands for the Lee-Yang-Parr correlation functional (B3LYP) (see $[24,25])$. This functional was combined with a quite large basis set, i.e., $6-311++G(2 d, 2 p) .6-311++G(2 d, 2 p)$ stands for a split-valance triple zeta $(\xi)$ enlarged with two diffuse basis functionals $(++)$, one is sp-orbitals added for the carbon atoms and s-orbital added to all hydrogen atoms. Additionally, larger polarization functionals, $2 d-$ and $2 p-$ orbitals added for the carbon and hydrogen atoms, respectively, were included. The frequency calculations were performed on all optimized geometries, and the absence of negative frequencies implies that the geometries are all minima points. Optimization and frequency calculations were performed using Gaussian 09 (see [5]), and data were visualized using GaussView (version 5.0.8) (see [26]) programs. The chemical structures, optimized geometries, the distributions, and energies of the highest occupied molecular orbitals (HOMOs) and the lowest unoccupied molecular orbitals (LUMOs) and also the total densities mapped with electrostatic potentials (ESPMs) at isovalue $=0.2$ a.u. are all included in Figure 3.
The ESPM is referring to a three-dimensional plot of the total electronic densities mapped with electrostatic potentials. Therefore, it helps in visualizing the electron density distribution around each atom/region of the molecule. The five isomers energies are all large negative values which confirm on the suitability of the applied level of theory. The five isomers can be arranged according to their total electronic energies and thus to their stability as follows: 2,3dimethylbutane $<3$-methylpentane $<2$,2-dimethylbutane $<2$-methylpentane $<$ hexane. By Figure 3, the energies of highest occupied molecular orbitals (HOMOs) and the lowest unoccupied molecular orbitals (LUMOs) are all negative and arranged the isomers in terms of their ability to donate/accept electrons during a chemical reaction.

The molecular graph of hexane and its isomers is shown in Figure 4. In this figure, while the vertices represent the atoms, the edges represent the chemical bond. We should note that the hydrogen atom is omitted.

Theorem 11. The first locating and multiplicative indices of Hexane are 222 and 1712237725, respectively. The second locating and multiplicative indices of Hexane are 140 and 12390400, respectively.

Proof. By taking into account Figure 4(a), let us first compute $\vec{v}_{i}$ for each $v_{i} \in V$ (hexane). Thus, we have

$$
\begin{aligned}
& \overrightarrow{v_{1}}=(0,1,2,3,4,5), \\
& \overrightarrow{v_{2}}=(1,0,1,2,3,4), \\
& \overrightarrow{v_{3}}=(2,1,0,1,2,3), \\
& \overrightarrow{v_{4}}=(3,2,1,0,1,2), \\
& \overrightarrow{v_{5}}=(4,3,2,1,0,1), \\
& \overrightarrow{v_{6}}=(5,4,3,2,1,0) .
\end{aligned}
$$

Then, by using equations (47) and (1), the first locating and multiplicative indices of hexane are presented by 


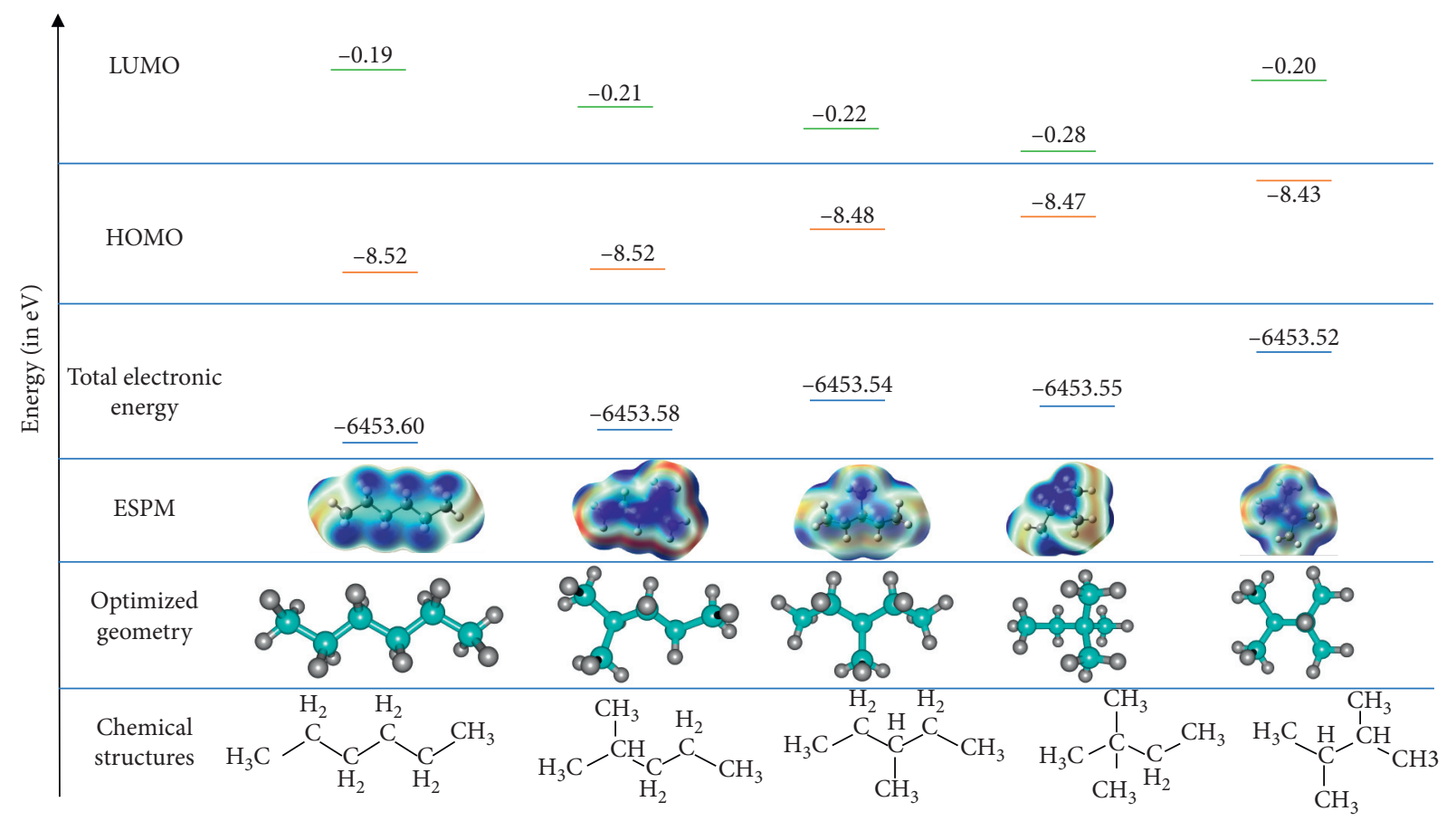

Figure 3: The chemical structures, optimized geometries, the distributions, and energies of the highest occupied molecular orbitals (HOMOs) and the lowest unoccupied molecular orbitals (LUMOs) and also the total densities mapped with electrostatic potentials (ESPMs) at isovalue $=0.2$ a.u. of (a) hexane, (b) 2-methylpentane, (c) 3-methylpentane, (d) 2,2-dimethylbutane, and (e) 2,3-dimethylbutane.

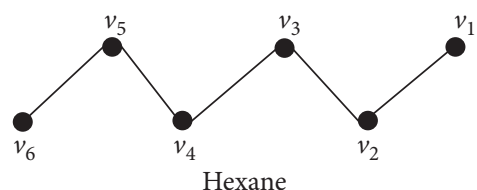

(a)

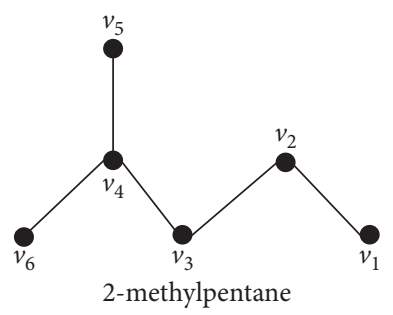

(b)

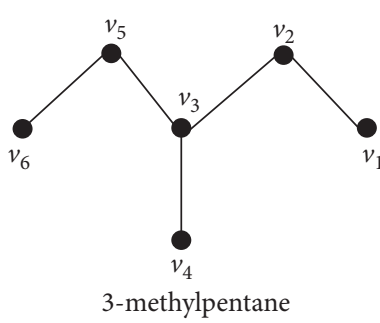

(c)

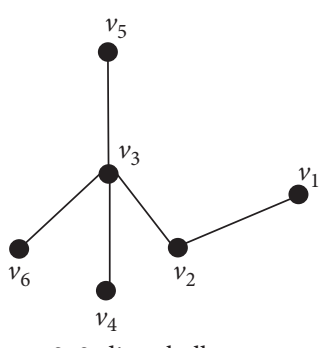

2, 2-dimethylbutane

(d)

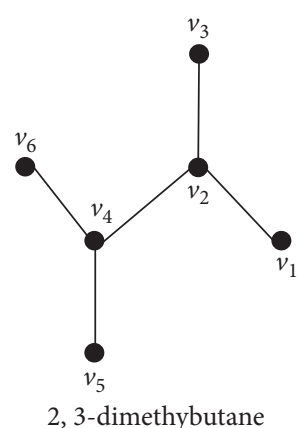

(e)

FIgURE 4: Molecular graphs of hexane and its isomers: (a) hexane; (b) 2-methylpentane; (c) 3-methylpentane; (d) 2,2-dimethylbutane; (e) 2,3-dimethylbutane. 


$$
\begin{aligned}
& M_{1}^{\mathscr{L}} \text { (hexane) }=\sum_{v_{i} \in V}\left(\vec{v}_{i}\right)^{2}=55+31+31+19+31+55=222, \\
& \prod_{1}^{\mathscr{L}}(\text { hexane })=\prod_{v_{i} \in V}\left(\vec{v}_{i}\right)^{2}=55 \times 31 \times 31 \times 19 \times 31 \times 55=1712237725 .
\end{aligned}
$$

On the other hand, by using equations (48) and (2), the second locating and multiplicative indices of hexane are presented by

$$
\begin{aligned}
M_{2}^{\mathscr{L}} \text { (hexane) } & =\sum_{v_{i} v_{j} \in E} \overrightarrow{v_{i}} \cdot \overrightarrow{v_{j}}=\left(\overrightarrow{v_{1}} \cdot \overrightarrow{v_{2}}\right)+\left(\overrightarrow{v_{2}} \cdot \overrightarrow{v_{3}}\right)+\left(\overrightarrow{v_{3}} \cdot \overrightarrow{v_{4}}\right)+\left(\overrightarrow{v_{4}} \cdot \overrightarrow{v_{5}}\right)+\left(\overrightarrow{v_{5}} \cdot \overrightarrow{v_{6}}\right) \\
& =40+22+16+22+40=140, \\
\prod_{2}^{\mathscr{L}}(\text { hexane }) & =\prod_{v_{i} v_{j} \in E} \overrightarrow{v_{i}} \cdot \overrightarrow{v_{j}}=\left(\overrightarrow{v_{1}} \cdot \overrightarrow{v_{2}}\right) \times\left(\overrightarrow{v_{2}} \cdot \overrightarrow{v_{3}}\right) \times\left(\overrightarrow{v_{3}} \cdot \overrightarrow{v_{4}}\right) \times\left(\overrightarrow{v_{4}} \cdot \overrightarrow{v_{5}}\right) \times\left(\overrightarrow{v_{5}} \cdot \overrightarrow{v_{6}}\right)=12390400 .
\end{aligned}
$$

Hence, the result is obtained.

In the following results, although we will follow completely the same way as in the proof of Theorem 11, we prefer to write some of those proofs again separately since the classification structural isomers is so important.

Theorem 12. The first locating and multiplicative indices of 2-methylpentane are 168 and 285874176, respectively. The second locating and multiplicative indices of 2-methylpentane are 94 and 2044416, respectively.

Proof. Considering Figure 4(b), let us calculate $\vec{v}_{i}$ for each $v_{i} \in V(2-$ methylpentane $)$. So, we have

$$
\begin{aligned}
& \overrightarrow{v_{1}}=(0,1,2,3,4,4), \\
& \overrightarrow{v_{2}}=(1,0,1,2,3,3), \\
& \overrightarrow{v_{3}}=(2,1,0,1,2,2), \\
& \overrightarrow{v_{4}}=(3,2,1,0,1,1), \\
& \overrightarrow{v_{5}}=(4,3,2,1,0,2), \\
& \overrightarrow{v_{6}}=(4,3,2,1,2,0) .
\end{aligned}
$$

By equations (47) and (1), the first locating and multiplicative indices of 2-methylpentane are given by

$$
\begin{aligned}
& M_{1}^{\mathscr{L}}(2-\text { methylpentane })=\sum_{v_{i} \in V}\left(\vec{v}_{i}\right)^{2}=46+24+14+16+34+34=168, \\
& \prod_{1}^{\mathscr{L}}(2-\text { methylpentane })=\prod_{v_{i} \in V}\left(\vec{v}_{i}\right)^{2}=46 \times 24 \times 14 \times 16 \times 34 \times 34=285874176 .
\end{aligned}
$$

Similarly as previous proofs, by equations (48) and (2), the second locating and multiplicative indices of 2-methylpentane are given by

$$
\begin{aligned}
M_{2}^{\mathscr{L}}(2-\text { methylpentane }) & =\sum_{v_{i} v_{j} \in E} \overrightarrow{v_{i}} \cdot \overrightarrow{v_{j}}=\left(\overrightarrow{v_{1}} \cdot \overrightarrow{v_{2}}\right)+\left(\overrightarrow{v_{2}} \cdot \overrightarrow{v_{3}}\right)+\left(\overrightarrow{v_{3}} \cdot \overrightarrow{v_{4}}\right)+\left(\overrightarrow{v_{4}} \cdot \overrightarrow{v_{5}}\right)+\left(\overrightarrow{v_{4}} \cdot \overrightarrow{v_{6}}\right) \\
& =22+16+12+22+22=94
\end{aligned}
$$


These all above progresses complete the proof.

Theorem 13. The first locating and multiplicative indices of 3-methylpentane are 118 and 163077057, respectively. The second locating and multiplicative indices of 3-methylpentane are 92 and 1557504, respectively.

Proof. By taking into account Figure 4(c), we compute $\vec{v}_{i}$ for each $v_{i} \in V$ (3-methylpentane), then we get

$$
\begin{aligned}
& \overrightarrow{v_{1}}=(0,1,2,3,3,4), \\
& \overrightarrow{v_{2}}=(1,0,1,2,2,3), \\
& \overrightarrow{v_{3}}=(2,1,0,1,1,2), \\
& \overrightarrow{v_{4}}=(3,2,1,0,2,3), \\
& \overrightarrow{v_{5}}=(3,2,1,2,0,1), \\
& \overrightarrow{v_{6}}=(4,3,2,3,1,0) .
\end{aligned}
$$

Similarly as previous proofs, by equations (47) and (1), the first locating and multiplicative indices of 3-methylpentane are given by

$$
\begin{aligned}
& M_{1}^{\mathscr{L}}(3-\text { methylpentane })=\sum_{v_{i} \in V}\left(\vec{v}_{i}\right)^{2}=39+19+11+27+19+39=118, \\
& \prod_{1}^{\mathscr{L}}(3-\text { methylpentane })=\prod_{v_{i} \in V}\left(\vec{v}_{i}\right)^{2}=39 \times 19 \times 11 \times 27 \times 19 \times 39=163077057 .
\end{aligned}
$$

By equations (48) and (2), the second locating and multiplicative indices of 3-methylpentane are given by

$$
\begin{aligned}
& M_{2}^{\mathscr{L}}(3-\text { methylpentane })=\sum_{v_{i} v_{j} \in E} \overrightarrow{v_{i}} \cdot \overrightarrow{v_{j}}=\left(\overrightarrow{v_{1}} \cdot \overrightarrow{v_{2}}\right)+\left(\overrightarrow{v_{2}} \cdot \overrightarrow{v_{3}}\right)+\left(\overrightarrow{v_{3}} \cdot \overrightarrow{v_{4}}\right)+\left(\overrightarrow{v_{3}} \cdot \overrightarrow{v_{5}}\right)+\left(\overrightarrow{v_{5}} \cdot \overrightarrow{v_{6}}\right)=26+12+16+12+26=92, \\
& \prod_{2}^{\mathscr{L}}(3-\text { methylpentane })=\prod_{v_{i} v_{j} \in E} \overrightarrow{v_{i}} \cdot \overrightarrow{v_{j}}=\left(\overrightarrow{v_{1}} \cdot \overrightarrow{v_{2}}\right) \times\left(\overrightarrow{v_{2}} \cdot \overrightarrow{v_{3}}\right) \times\left(\overrightarrow{v_{3}} \cdot \overrightarrow{v_{4}}\right) \times\left(\overrightarrow{v_{3}} \cdot \overrightarrow{v_{5}}\right) \times\left(\overrightarrow{v_{5}} \cdot \overrightarrow{v_{6}}\right)=26 \times 12 \times 16 \times 12 \times 26=1557504 .
\end{aligned}
$$

These all above progresses complete the proof.

Theorem 14. The first locating and multiplicative indices of 2,2-dimethylbutane are 120 and 38162432, respectively. The second locating and multiplicative indices of 2,2-dimethylbutane are 65 and 290304, respectively.

Proof. By Figure 4(d), for $1 \leq i \leq 3$, the vectors $\vec{v}_{i}$ for each $v_{i} \in V$ (2,2-dimethylbutane) can be obtained as

$$
\begin{aligned}
& \overrightarrow{v_{1}}=(0,1,2,3,3,3), \\
& \overrightarrow{v_{2}}=(1,0,1,2,2,2), \\
& \overrightarrow{v_{3}}=(2,1,0,1,1,1), \\
& \overrightarrow{v_{4}}=(3,2,1,0,2,2), \\
& \overrightarrow{v_{5}}=(3,2,1,2,0,2), \\
& \overrightarrow{v_{6}}=(3,2,1,2,2,0) .
\end{aligned}
$$

Thus, as previously, by equations (47) and (1) and equations (48) and (2), we obtain the required results on the first and second locating and multiplicative locating indices for 2,2-dimethylbutane.

Finally, let us consider Figure 4(e). Then, the vectors $\vec{v}_{i}$ for each $v_{i} \in V$ (2,3-dimethylbutane) can be obtained as

$$
\begin{aligned}
& \overrightarrow{v_{1}}=(0,1,2,2,3,3), \\
& \overrightarrow{v_{2}}=(1,0,1,1,2,2), \\
& \overrightarrow{v_{3}}=(2,1,0,2,3,3), \\
& \overrightarrow{v_{4}}=(2,1,2,0,1,1), \\
& \overrightarrow{v_{5}}=(3,2,3,1,0,2), \\
& \overrightarrow{v_{6}}=(3,2,3,1,2,0),
\end{aligned}
$$

with the same approach as before, by equations (47) and (1) and equations (48) and (2), we get the next final theorem for 
TABLE 1: Hexane and its isomers with their physico-chemical properties and topological indices values.

\begin{tabular}{|c|c|c|c|c|c|c|c|c|}
\hline Isomer name & F.L.I & S.L.I & F.M.I & S.M.I & B.P. $\mathbf{F}^{\circ}$ & M.P. $\mathbf{F}^{\circ}$ & E.C./eV & F.P. \\
\hline Hexane & 222 & 140 & 1712237725 & 12390400 & 155.7 & -139.5 & -6448.22 & 247.2 \\
\hline 2-Methylpentane & 168 & 94 & 285874176 & 2044416 & 140.5 & -244.7 & -6448.21 & 266 \\
\hline 3-Methylpentane & 118 & 92 & 163077057 & 1557504 & 145.9 & -180.4 & -6448.16 & 266 \\
\hline 2,2-Dimethylbutane & 120 & 65 & 38162432 & 290304 & 121.5 & -147.6 & -6448.20 & 244 \\
\hline 2,3-Dimethylbutane & 130 & 72 & 64304361 & 524288 & 136.4 & -199.5 & -6448.15 & 244 \\
\hline
\end{tabular}

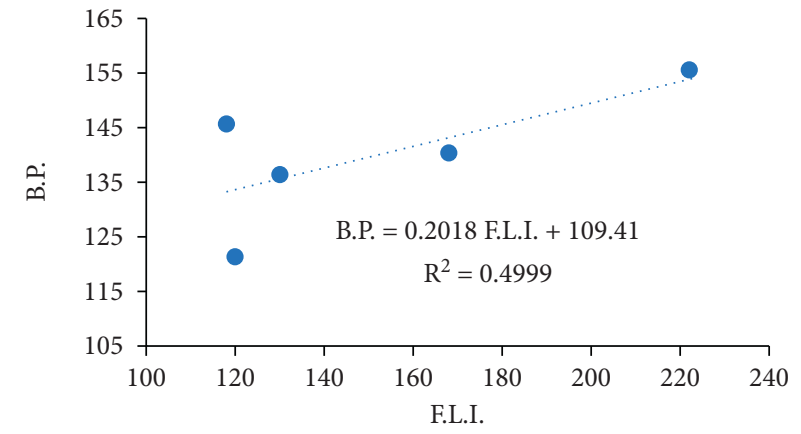

F.L.I.

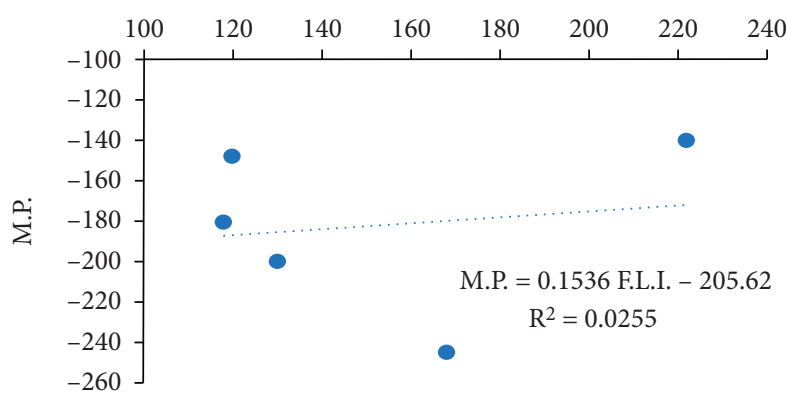

F.L.I.
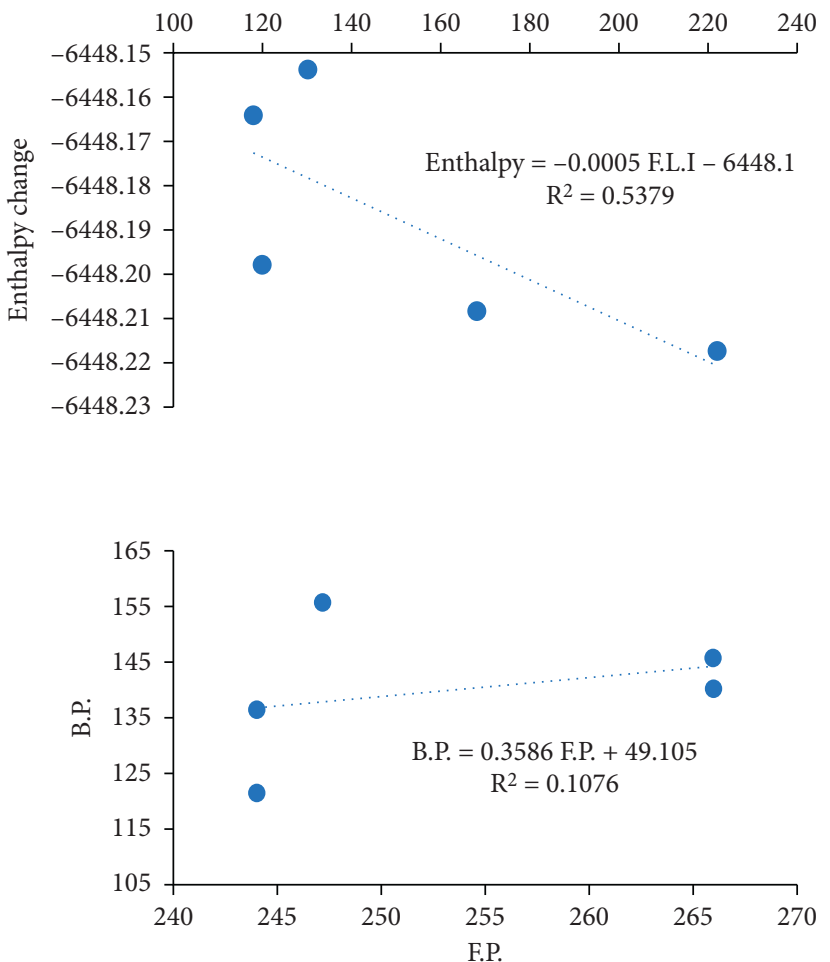

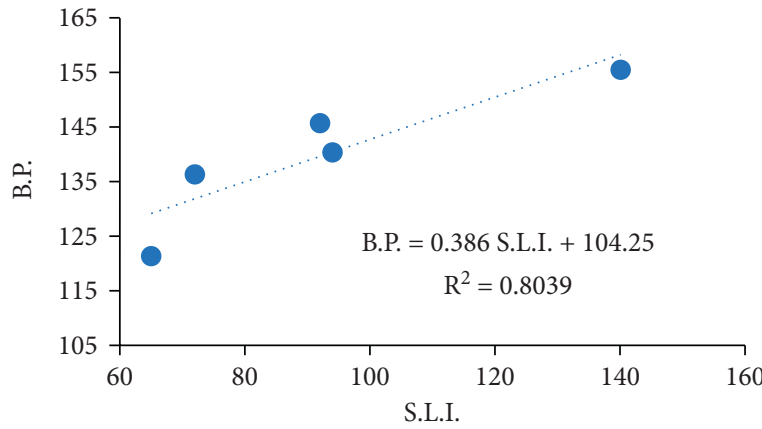

S.L.I.
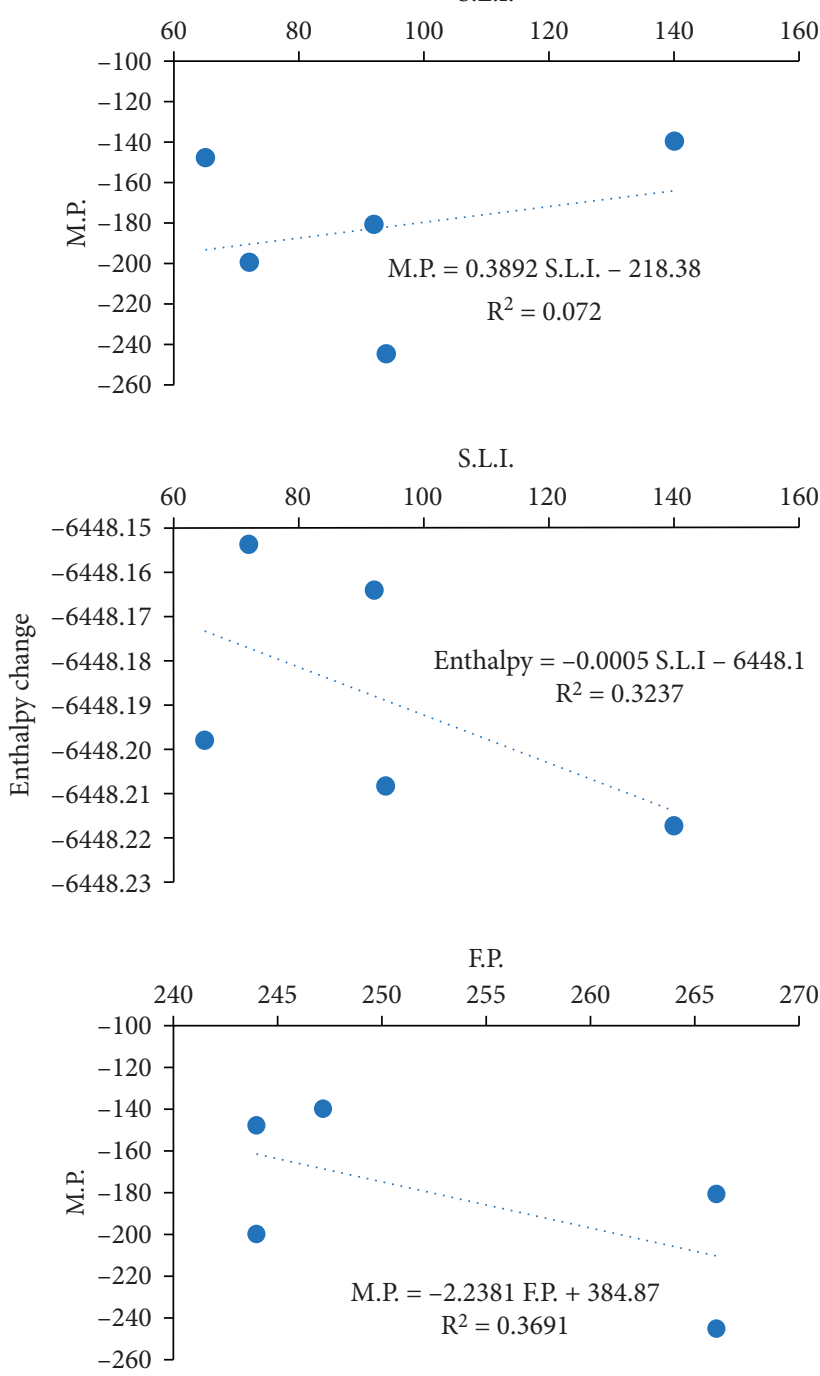

FIGURe 5: Graphical relationships between the boiling points (B.P.), melting points (M.P.), flash points (F.P.), enthalpy change (E.G.), and the calculated topological indices of hexane and its four isomers, where R2 represents the correlation coefficient. 
the first and second locating and multiplicative indices over 2,3-dimethylbutane.

Theorem 15. The first locating and multiplicative indices of 2,3-dimethylbutane are 130 and 64304361, respectively. The second locating and multiplicative indices of 2,3-dimethylbutane are 72 and 524288, respectively.

Table 1 indicates the exact values of first and second locating and multiplicative locating indices of hexane and its isomers with their physico-chemical properties such as boiling point (B.P.), melting point (M.P.), enthalpy change (E.C.), and flash point (F.P.).

Figure 5 indicates how much the obtained topological indices are correlated with the well-known physiochemical properties, i.e., the five investigated isomers. The degree of correlation between any two data sets is measured by the value of the correlation coefficient $\left(R^{2}\right)$. When the value of $R^{2}$ becomes close to unity, two data sets are more correlated. We can also note from Figure 5 that $R^{2}$ of the plot between F.L.I and boiling points (B.P.) equals 0.458 while it is equal to 0.781 for the plot between S.L.I and boiling points. In fact these two obtained values of $R^{2}$ for these two plots are quite satisfactory. Similar conclusion can be obtained for the plots among F.L.I and S.L.I data, and the enthalpy changes values since $R^{2}$ equals 0.538 and 0.324 for these two plots, respectively. The values of $R^{2}$ are not big enough but still indicates good correlations between these two data sets. However, the achieved correlation coefficients between two topological indices and the melting points of five isomers are too small and so should be indicated a poor correlation between them since the values of $R^{2}$ in these two plots are less than 0.2 . The plots between F.L.I and the flash points (F.P.) are equal to 0.108 while a better correlation is obtained between S.L.I and F.P. as the value $R^{2}=0.369$. Therefore, the former plot represents a poor correlation and the later can be considered as a better correlation.

\section{Conclusion}

This study combined pure data from the chemistry textbooks and a mathematical effort to find new topological indices of five well-known chemical compounds. The cases in which good correlations were obtained suggested the validity of the calculated topological indices to be further used to predict the physio-chemical properties of much complicated chemical compounds.

\section{Data Availability}

The chemical data used in this paper are strictly personal since most of those are obtained with some payments in a computing center after the theoretical parts obtained. However, the reader may contact the corresponding author for more details and special permissions of data.

\section{Conflicts of Interest}

The authors declare that they have no conflicts of interest.

\section{Acknowledgments}

This work was funded by the Deanship of Scientific Research (DSR), King Abdulaziz University, Jeddah, under grant no. G: 218-247-1442. The authors, therefore, acknowledge DSR technical and financial support. The authors acknowledge Prof. Nuha Wazzan from Chemistry department at King Abdulaziz University for her contribution with the DFT calculations and King Abdulaziz University's High-Performance Computing Centre (Aziz Supercomputer) (http:// hpc.kau.edu.sa) for supporting the computation for the work described in this paper.

\section{References}

[1] S. Aykaç, N. Akgüneş, and A. S. Çevik, "Analysis of Zagreb indices over zero-divisor graphs of commutative rings," Asian-European Journal of Mathematics, vol. 12, no. 06, Article ID 2040003, 2019.

[2] K. C. Das, "On the Zagreb energy and Zagreb estrada index of graphs, MATCH commun," Math. Comput. Chem, vol. 82, no. 2, pp. 529-542, 2019.

[3] M. V. Diudea, M. S. Florescu, and P. V. Khadikar, Molecular Topolgy and its Applications, Eficon, Bucarest, Romania, 2006.

[4] M. V. Diudea, Nanomolecules and Nanostructures-Poynomial and Indices, University Kragujevac, Kragujevac, Serbia, 2010.

[5] M. J. Frisch, Gaussian 09 Programmer's Reference, Gaussian: 2009.

[6] I. Gutman and B. Furula, Eds., Distance in Molecular Graphs, University Kragujevac, Kragujevac, Serbia, 2012.

[7] M. Karelson, Molecular Descriptors in QSAR-QSPR, Wiley, New York, NY, USA, 2000.

[8] R. Todeschini and V. Consonni, Molecular Descriptors for Chemoinformatics, Wiley VCH, Weinheim, Germany, 2009.

[9] I. Gutman and N. Trinajstić, "Graph theory and molecular orbitals. Total $\varphi$-electron energy of alternant hydrocarbons," Chemical Physics Letters, vol. 17, no. 4, pp. 535-538, 1972.

[10] A. Alwardi, A. Alqesmah, R. Rangarajan, and I. N. Cangul, "Entire Zagreb indices of graphs," Discrete Mathematics, Algorithms and Applications, vol. 10, no. 03, 16 pages, Article ID 1850037, 2018.

[11] K. C. Das, A. Yurttas, M. Togan, A. S. Cevik, and I. N. Cangul, "The multiplicative Zagreb indices of graph operations," Journal of Inequalities and Applications, vol. 90, 2013.

[12] K. C. Das, N. Akgunes, M. Togan, A. Yurttas, I. N. Cangul, and A. S. Cevik, "On the first Zagreb index and multiplicative Zagreb coindices of graphs," Analele stiintifice ale Universitatii Ovidius Constanta, vol. 24, 2016.

[13] I. Gutman and K. C. Das, "The first Zagreb index 30 years after, MATCH Commun," Math. Comput. Chem., vol. 50, pp. 83-92, 2004.

[14] M. H. Khalifeh, H. Yousefi-Azari, and A. R. Ashrafi, "The first and second Zagreb indices of some graph operations," Discrete Applied Mathematics, vol. 157, no. 4, pp. 804-811, 2009.

[15] S. Nikolić, G. Kovac̃ević, A. Milic̃ević, and N. Trinajstić, "The Zagreb indices 30 years after," Croatica Chemica Acta, vol. 76, pp. 113-124, 2003.

[16] A. Yurttas, M. Togan, V. Lokesha, I. N. Cangul, and I. Gutman, "Inverse problems for Zagreb indices," Journal of Mathematical Chemistry, vol. 57, pp. 609-615, 2019.

[17] B. Zhou and I. Gutman, "Further properties of Zagreb indices," MATCH Communications in Mathematical and in Computer Chemistry, vol. 54, pp. 233-239, 2005. 
[18] S. A. Wazzan, "Topological indices of Parkinson's disease drug carbidopa," Far East Journal of Mathematical Sciences (FJMS), vol. 118, no. 1, pp. 45-62, 2019.

[19] S. Wazzan, "Some new topological indices of aspirin," Proceedings of the Jangjeon Mathematical Society, vol. 23, no. 1, pp. 113-124, 2019.

[20] S. A. Wazzan and A. Saleh, "On the first and second locating Zagreb indices of graphs," Applied Mathematics, vol. 10, no. 10, pp. 805-816, 2019.

[21] H. N. Ramaswamy, A. Alwardi, and N. R. Kumar, "On the locating matrix of a graph and its spectral analysis," Computer Science Journal of Moldova, vol. 25, no. 3, 75 pages, 2017.

[22] G. Chartand and L. Lesniak, Graphs and Digraphs, CRC Press, Boca Raton, FL, USA, 4 edition, 2005.

[23] J. Li, J.-M. Guo, and W. C. Shiu, "On the second largest laplacian eigenvalues of graphs," Linear Algebra and Its Applications, vol. 438, no. 5, pp. 2438-2446, 2013.

[24] A. D. Becke, "Density-functional exchange-energy approximation with correct asymptotic behavior," Physical Review A, vol. 38, no. 6, pp. 3098-3100, 1988.

[25] C. Lee, W. Yang, and R. G. Parr, "Development of the ColleSalvetti correlation-energy formula into a functional of the electron density," Physical Review B, vol. 37, no. 2, pp. 785-789, 1988.

[26] R. Dennington, T. Keith, and J. Millam, Gauss View, Semichem Inc., Shawnee, KS, USA, 2009. 